\title{
NATURAL CONVECTION FROM FOUR CIRCULAR CYLINDERS IN ACROSS ARRANGEMENT WITHIN HORIZONTAL ANNULAR SPACE
}

\author{
Houssem LAIDOUDI \\ "Laboratory of Sciences and Marine Engineering, Faculty of Mechanical Engineering, \\ USTO-MB, BP 1505, El-Menaouer, Oran, 31000, Algeria \\ houssem.laidoudi@univ-usto.dz
}

received 18 April 2020, revised 24 June 2020, accepted 26 June 2020

\begin{abstract}
Numerical investigation is accomplished to study the roles of governing parameters of natural convection on the fluid motion and heat transfer rate of four heated circular cylinders placed inside a circular enclosure of cold surface. The cylinders are positioned in across arrangement. The representative results are obtained within the ranges of initial conditions as: Prandtl number $(\operatorname{Pr}=7.1$ to 1000) and Rayleigh number $\left(\mathrm{Ra}=10^{3}\right.$ to $\left.10^{5}\right)$. The average Nusselt number of each inner cylinder is computed. The effects of thermal buoyancy strength on the fluid motion and temperature are also illustrated. It was found that the heat transfer rate of cylinders depends significantly on the position inside the enclosure. Moreover, the role of Prandtl number on flow and thermal patterns is negligible. The values of Nusselt number are also given, which can be useful for some engineering applications.
\end{abstract}

Key words: Natural convection, multiple cylinders, annular space, heat transfer, numerical simulation

\section{INTRODUCTION}

The heat transfer inside an annular space is an important phenomenon that can be widely encountered in many industrial applications such as heat exchangers, refineries, cooling small electronic devices, marine engineering applications, chemical processes and so on. Therefore, an important number of scientific researches have been devoted recently to understand the governing parameters on the fluid flow and thermal patterns. For example, Masoumi et al. (2019) studied the laminar natural convection between two horizontal cylinders. The cylinders were placed in a concentric manner. The fluid used for this work was yield stress fluid. The governing parameters of this research were limited for $\mathrm{Ra}=10^{3}$ to $10^{6}$. Pandey et al. (2019) presented an extensive review of the roles of thermo-physical characteristics of the fluid and the geometrical forms of the inner bodies on natural convection inside square cavity. The studied characteristics are modulated by the dimensionless numbers of Rayleigh and Prandtl. Aly (2017) investigated the natural convection heat transfer from two circular cylinders placed in tandem arrangement within rectangular cavity. The annular space for this research was assumed to be porous. The research examined the porous characteristics and the cylinder dimensions on the rate of heat transfer. Hussein (2013) numerically examined the effects of geometrical modifications of outer square cavity on the heat transfer rate of circular cylinder placed in the centre of this outer cavity.

It can be seen that the heat transfer in annular space depends expensively on the geometrical configuration of heated or cold organs and the thermo-characteristics of the fluid. For this reason, several researchers have worked on these aspects in order to enhance the heat transfer rate in annular configurations.

Matin and Khan (2013) numerically studied the thermal buoy- ancy of natural convection in annular space. The studied geometry consisted of two horizontal concentric cylinders of circular shape. The computed domain was assumed to be filled with nonNewtonian power fluids. The controlling parameters of Prandtl and Rayleigh numbers were studied for $\mathrm{Pr}=0.7$ to 1000 and $\mathrm{Ra}=10^{3}$ to $10^{5}$. The results showed that the first category of power-law fluids (shear thinning) having a tendency to improve the rate of heat transfer. On the other hand, the shear thickening fluids reduced the heat transfer rate. Abu-Nada et al. (2008) for the same the geometry of Matin and Khan (2013) used nanofluids instead of base-fluid. They confirmed that the nanofluids enhance the thermo-physical characteristics of base-fluid, and consequently, improve the heat transfer rate. Nada and Said (2019) found that there is a possibility to increase the heat transfer rate of single cylinder placed within cold outer cylinder. The solution was added to some radial fins to the wall of inner cylinder. El-Maghlany et al. (2016), Char and Lee (1998) and Nasiri et al. (2017) used the nanofluids as convective medium to transfer heat between two horizontal cylinders. The cylinders were arranged in an eccentric manner. They found that the eccentricity factor of inner cylinder increases the heat transfer rate. Iqbal et al. (2017), Arbaban and Salimpour (2014), Ha et al. (2004), Hadidi et al. (2020) also used the same idea to increase the effect of heat transfer. Laidoudi (2020) studied the natural convection in enclosed cold cavity in which two hot circular cylinders were placed in tandem. Laidoudi et al. (2020) also studied a numerical investigation on natural convection within an annular space of a special shape of horizontal inner cylinder. It was found that the thermo-physical proprieties of fluids have a limited effect on convective heat transfer. Eid (2011) performed an experimental study on free convection in annulus of elliptical cross-section. Kozlov (2018) numerically examined the effect of rotational vibrations on flow and thermal behaviours in annulus. Sheikhzadeh et al. (2013) numerically 
combined the effects of radial fins of inner cylinder with nanofluid characteristics to improve the heat transfer in an annulus of circular form.

From these mentioned papers and others such as Zhang et al. (1992), Kuehn and Goldstein (1976) and Ho et al. (1989), the analyses of fluid flow inside the annulus showed that the apparition of two loops and the form of these loops as well as their main centre depend mainly on the value of Rayleigh number, that is, the thermal buoyancy strength.

After this presentation of previous researches on laminar natural convection in annular space, it can be concluded that the heat transfer rate of inner cylinders is significantly affected by the shapes of the domain and thermo-characteristics of fluid. Although there are many researches that cover the buoyancy-driven flow in annular space, few papers studied the multiple arranged cylinders in circular enclosure under the effect of thermal buoyancy. For that reason, the present paper is an attempt to add some insights about the effects of mentioned parameters on the natural convection heat transfer from four circular cylinders placed in across arrangement within cold circular cylinder. The results are analysed and interpreted for the range of the following parameters: $\mathrm{Ra}=$ $10^{3}$ to $10^{5}$ and $\operatorname{Pr}=7.1$ to 1000 . The evolutions of heat transfer rate are quantified by the value of average Nusselt number.

\section{PHYSICAL DOMAIN AND MATHEMATICAL MODELLING}

The studied problem is schematized in Fig. 1. It involves a cold circular enclosure $\left(T_{c}\right)$ of a diameter $H$. Inside this circular cavity, four circular cylinders are placed in across arrangement. The cylinders are hot $\left(T_{h}\right)$ and they have a similar diameter (d). This diameter $(d)$ is defined by the blockage ratio $B=d / H=0.2$. The gap distance between the centres of parallel cylinders is given by the value $S=0.5 \mathrm{H}$. Effectively, the studied domain is a symmetrical arrangement. The annular space between inner cylinders and the outer cavity is assumed to be filled with incompressible Newtonian fluid. Due to temperature difference between the surfaces of inner cylinders and outer cylinder that inducesthermal buoyancy force, which is the source of fluid motion.

These kinds of physical phenomena are mostly modelled by the governing equations of continuity, Navier-Stockes and energy. And the effect of buoyancy-driven flow is treated by Bousssinesq approximation.

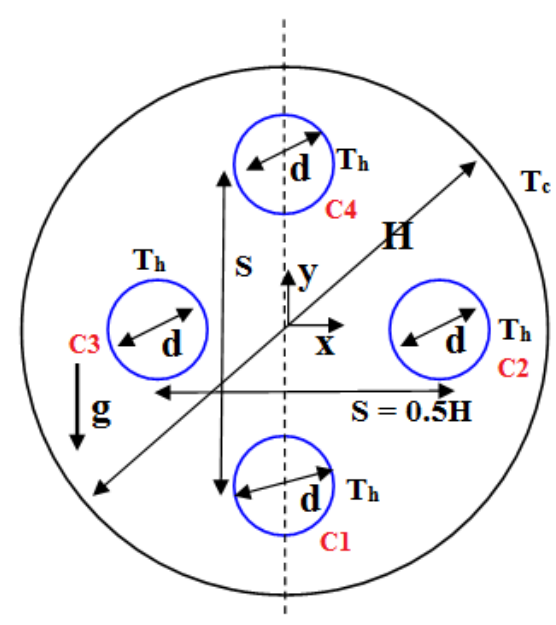

Fig. 1. Schematic view of studied geometry
The governing equations for tow-dimensional flow in Cartesian coordinate systems can be written in dimensionless form as:

$\frac{\partial u^{\bullet}}{\partial x^{\bullet}}+\frac{\partial v^{\bullet}}{\partial y^{\bullet}}=0(1)$

$u^{\bullet} \frac{\partial u^{\bullet}}{\partial x^{\bullet}}+v^{\bullet} \frac{\partial u^{\bullet}}{\partial y^{\bullet}}=-\frac{\partial p^{\bullet}}{\partial x^{\bullet}}+\operatorname{Pr}\left(\frac{\partial^{2} u^{\bullet}}{\partial x^{\bullet 2}}+\frac{\partial^{2} u^{\bullet}}{\partial y^{\bullet}}\right)(2)$

$u^{\bullet} \frac{\partial v^{\bullet}}{\partial x^{\bullet}}+v^{\bullet} \frac{\partial v^{\bullet}}{\partial y^{\bullet}}=-\frac{\partial p^{\bullet}}{\partial y^{\bullet}}+\operatorname{Pr}\left(\frac{\partial^{2} v^{\bullet}}{\partial x^{\bullet 2}}+\frac{\partial^{2} v^{\bullet}}{\partial y^{\bullet 2}}\right)+R a \cdot \operatorname{Pr} . T^{\bullet}(3)$

$u^{\bullet} \frac{\partial T^{\bullet}}{\partial x^{\bullet}}+v^{\bullet} \frac{\partial T^{\bullet}}{\partial y^{\bullet}}=\left(\frac{\partial^{2} T^{\bullet}}{\partial x^{\bullet 2}}+\frac{\partial^{2} T^{\bullet}}{\partial y^{\bullet 2}}\right)(4)$

The number $\mathrm{Ra}$ and $\mathrm{Pr}$ are given by following expressions, respectively:

$R a=\frac{g \beta\left(T_{h}-T_{c}\right) d^{3}}{v \alpha}, \operatorname{Pr}=\frac{v}{\alpha}(5)$

The written variables in governing equations refer to: $u$ and $v$ are velocity components along the $x$ and $y$-directions, respectively; $p$ is the pressure; and $T$ is the temperature. These variables are written in dimensionless form as:

$x^{\bullet}=\frac{x}{d}, y^{\bullet}=\frac{y}{d}, u^{\bullet}=\frac{u d}{\alpha}, v^{\bullet}=\frac{v d}{\alpha}, p^{\bullet}=\frac{p d^{2}}{\rho \alpha^{2}}, T^{\bullet}=\frac{T-T_{C}}{T_{h}-T_{C}}(6)$

The studied fluid is defined by its thermo-physical characteristics, which are given by density $\rho$, kinematic viscosity $v$, thermal diffusivity $\alpha$ and the expansion coefficient $\beta$. However, $g$ refers to gravitational acceleration.

The average Nusselt number of each inner cylinder is calculated by integrating the local Nusselt number along the surface of the cylinder. The expressions for the Nusselt number are:

$N u_{l}=\left.\frac{\partial \phi}{\partial n}\right|_{\text {wall }}(7)$

$N u=\frac{1}{s} \int_{0}^{s} N u_{l} d s(8)$

where $n$ and $s$ are the direction normal to the wall.

Due to numerical considerations, appropriate boundary conditions are subjected to domain extremities as:

Around the outer cylinder; cold surface with no-slip boundary layer as:

$u^{\bullet}=0, v^{\bullet}=0, T^{\bullet}=0(9)$

On the surfaces of inner cylinders; hot surfaces with no-slip boundary layer as:

$u^{\bullet}=0, v^{\bullet}=0, T^{\bullet}=1(10)$

\section{GRID INDEPENDENCY AND VALIDATION TESTS}

The governing equations for present work are non-linear partial differential. Therefore, the suitable commercial code ANSYCFX is used to solve them numerically. The software transforms the equations into matrix system under the aspect of finite-volume method. The convective terms of matrix system is solved by High resolution discretization scheme. However, SIMPLEC (SemiImplicit Method for Pressure-Linked Equations-Consistent) is used for velocity-pressure coupling. The obtained results from these equations are considered when the relative error of the equations becomes less than $10^{-8}$ for continuity and momentum equations and $10^{-6}$ for energy equation. 
The grid for present geometry is meshed by using the software Gambit. The elements of grid are non-uniform and unstructured. The elements of the grid are concentrated near the walls of cylinders where thermal and flow layers are very thin. Fig. 2 shows the grid for present investigation. The grid contains 74,962 elements. This value was considered after examining the grid indecency test on obtained results. This step is obviously represented in the results of Tab. 1.

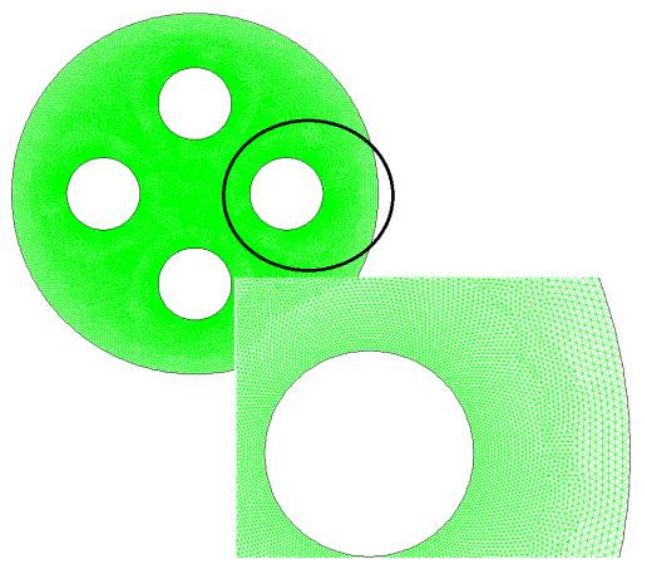

Fig. 2. Typical grid used for present investigation

Tab. 1. Results of grid independency test for $\mathrm{Pr}=10$ and $\mathrm{Ra}=10^{4}$

\begin{tabular}{|l|c|c|c|}
\hline Mesh & Elements & $\mathrm{Nu}(\mathrm{C} 1)$ & Variation (\%) \\
\hline M1 & 10324 & 3.79345 & 0.74 \\
\hline M2 & 32980 & 3.76674 & 0.25 \\
\hline M3 & 74962 & 3.7575 & - \\
\hline
\end{tabular}

This section also incorporates the validation test. This step is shown to prove the accuracy and precision of the used numerical method. To do so, we have executed the same geometry studied by Kuehn and Goldstein (1976) and Matin and Khan (2013). The comparison between all results is depicted in Fig. 3. In fact, Fig. 3 shows the variation of average Nusselt number versus Rayleigh number of a single circular cylinder placed at the centre of circular enclosure for $\mathrm{Pr}=1$. Fig. 3 shows a good agreement between all the results. The relative error between the present results and the previous works is almost less than $2 \%$.

\section{RESULT AND DISCUSSION}

The present investigation is conducted to understand the roles of Rayleigh number and Prandtl number on laminar natural convection in annular space. The studied domain consists of four equal-sized cylinders in across arrangement confined in circular enclosure. The inner cylinders are heated with constant temperature $\left(T_{h}\right)$ and the outer cylinder is kept cold with constant temperature $\left(T_{c}\right)$. The computational domain is assumed to be filled with incompressible Newtonian fluid. The circulation of flow inside the domain and temperature distributions are depicted as representative streamline and isotherm contours. However, the average Nusselt number is graphically plotted versus the studied parameters.

Fig. 4 and 5 shows the isotherms and streamlines inside the annular space with different values of Rayleigh and Prandtl num- bers. It can be noticed that the particles of the fluid around the heated cylinders become hot, and consequently, the fluid density in these regions becomes lighter, which leads to the movement of lighter regions towards the upper part of the cavity. On the other hand, the fluid particles by the curvature of cold cavity becomes heavier and they move down towards the lower part of circular enclosure. This successive movement of fluid generates two symmetrical loops in the annular space as it is showing Fig. 5 for $\mathrm{Ra}=10^{3}$.

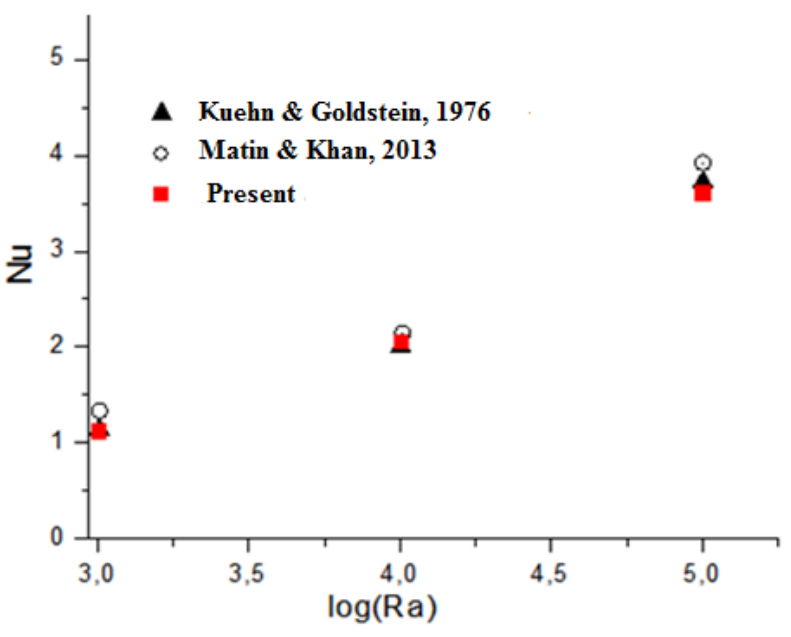

Fig. 3. Validation test
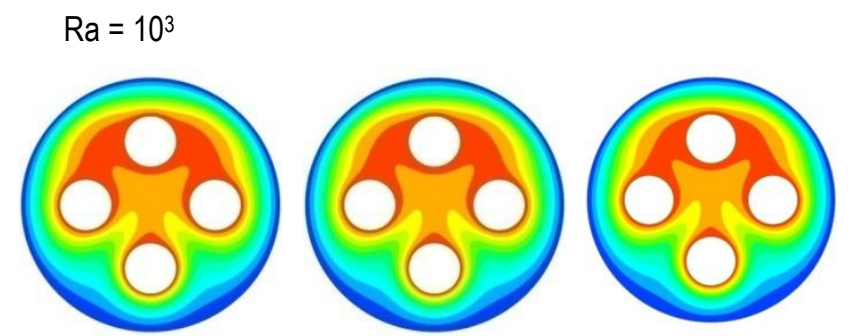

$\mathrm{Ra}=10^{4}$
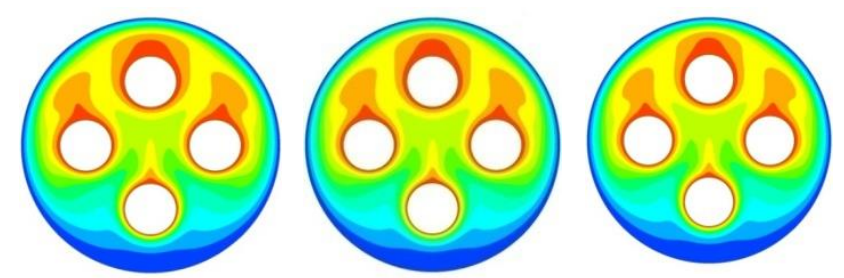

$\mathrm{Ra}=10^{5}$
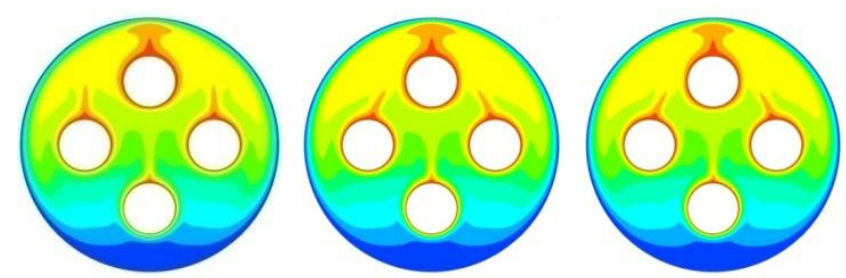

$\operatorname{Pr}=7.1$

$\mathrm{Pr}=50$

$\operatorname{Pr}=1000$

Fig. 4. Isotherm contours for three values of Prandtl and Rayleigh numbers 
It is also shown that an increase in the value of Rayleigh number leads to an increase in the strength of thermal buoyancy, and as a consequence, the centre of main loops shifts upwards. Moreover, the increase in the value of Rayleigh number creates a complicated flow inside the domain. Two extra small loops appear above the lower cylinder for $\mathrm{Ra}=10^{5}$. For all cylinders, the lower part of cylinder has remarkable isotherms in comparison to the upper part. These thin regions of isotherms indicate that the thermal gradient is very important, which results in an important rate of heat transfer in these zones. Furthermore, the isotherms show that lateral distributions of isotherms decrease with an increase in the Rayleigh number, hinting that the heat transfer of inner cylinders increases with increasing Rayleigh number. The influence of Prandtl number on streamlines and isotherms is almost negligible. Finally, it can be expected that the lower cylinder (C1) has the highest value of heat transfer rate, whereas the upper cylinder (C4) has the lowest value of heat transfer rate. Also, the plumes that are shown on cylinders show the direction of the flow.
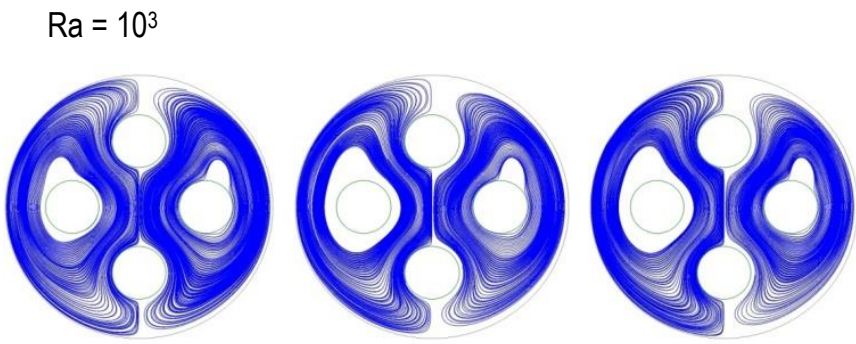

$\mathrm{Ra}=10^{4}$
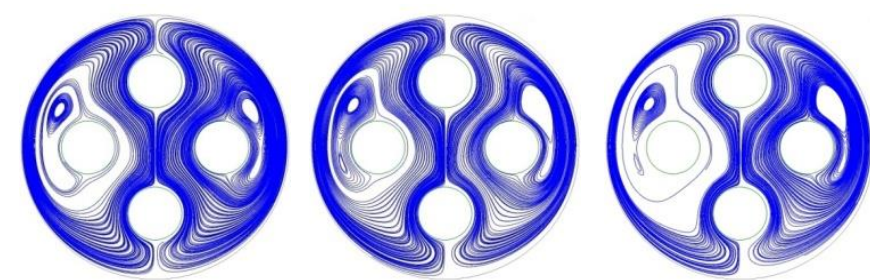

$\mathrm{Ra}=10^{5}$

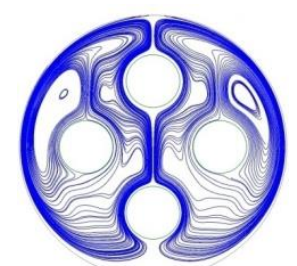

$\operatorname{Pr}=7.1$

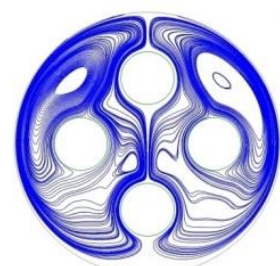

$\operatorname{Pr}=50$

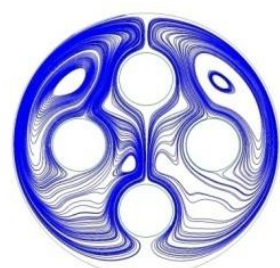

$\operatorname{Pr}=1000$
Fig. 5. Streamline contours for three values of Prandtl and Rayleigh numbers

In order to elucidate additionally the flow inside the annular space, Fig.6 depicts the evolution of dimensionless competent velocity profiles of $y$-direction along the line $x$ for $y=0$. The velocity profiles indicate that an increase in the value of Rayleigh number increases the velocity. Also, the effect of Prandtl number is almost negligible. The negative values of velocity are close to the outer cylinder, whereas, the positive values are in the vicinity of inner cylinders. The maximum value of velocity is induced by cold source.

Fig. 7 shows the average Nusselt number of inner cylinders as a function of Prandtl number and Rayleigh number. As it was expected, the lower cylinder has the highest values of Nusselt number. Generally, as we move from the lower cylinder (C1) to the upper cylinder (C4), the average Nusselt number decreases. Increase in Rayleigh number increases the average Nusselt number of all cylinders. On the other hand, with an increase in Prandt number, the values of Nusselt number remains constant for all cylinders.
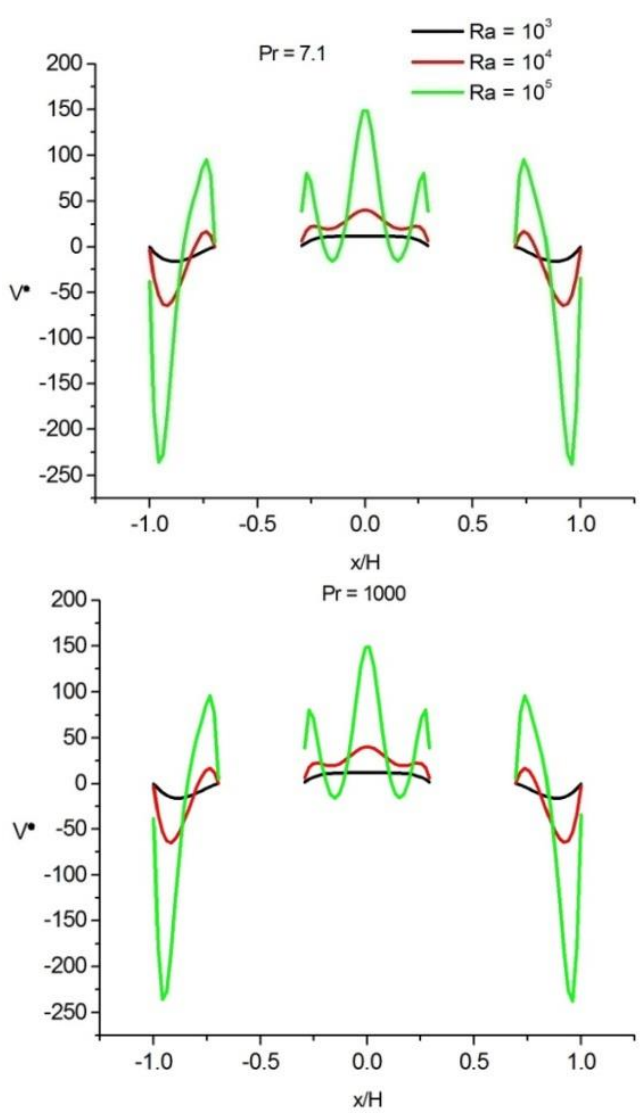

Fig. 6. Dimensionless velocity profiles along $x$-direction at $y=0$ for different values of $\mathrm{Ra}$ and $\mathrm{Pr}$

\section{CONCLUSION}

Numerical simulations are carried out to study the laminar natural convection in annular space. The domain consists of four circular cylinders, which are placed in across arrangement inside circular enclosure. The work tested the roles of Prandtl and Rayleigh numbers on the flow and thermal patterns. The obtained results of the present study let us draw some new points:

- The heat transfer rate of inner cylinders depends strongly on the position of cylinder.

- Increase in the value of Rayleigh number increases the heat transfer rate.

- The Prandtl number is a negligible parameter for the natural convection in annular space.

- As we move from the lower part to upper region of annular space increases the heat transfer rate.

- This arrangement of circular cylinders generate new extra loops above the lower cylinder.

- The upper part of inner cylinder has the lowest value of heat transfer rate, whereas, the bottom part of cylinder has the highest value of heat transfer. 

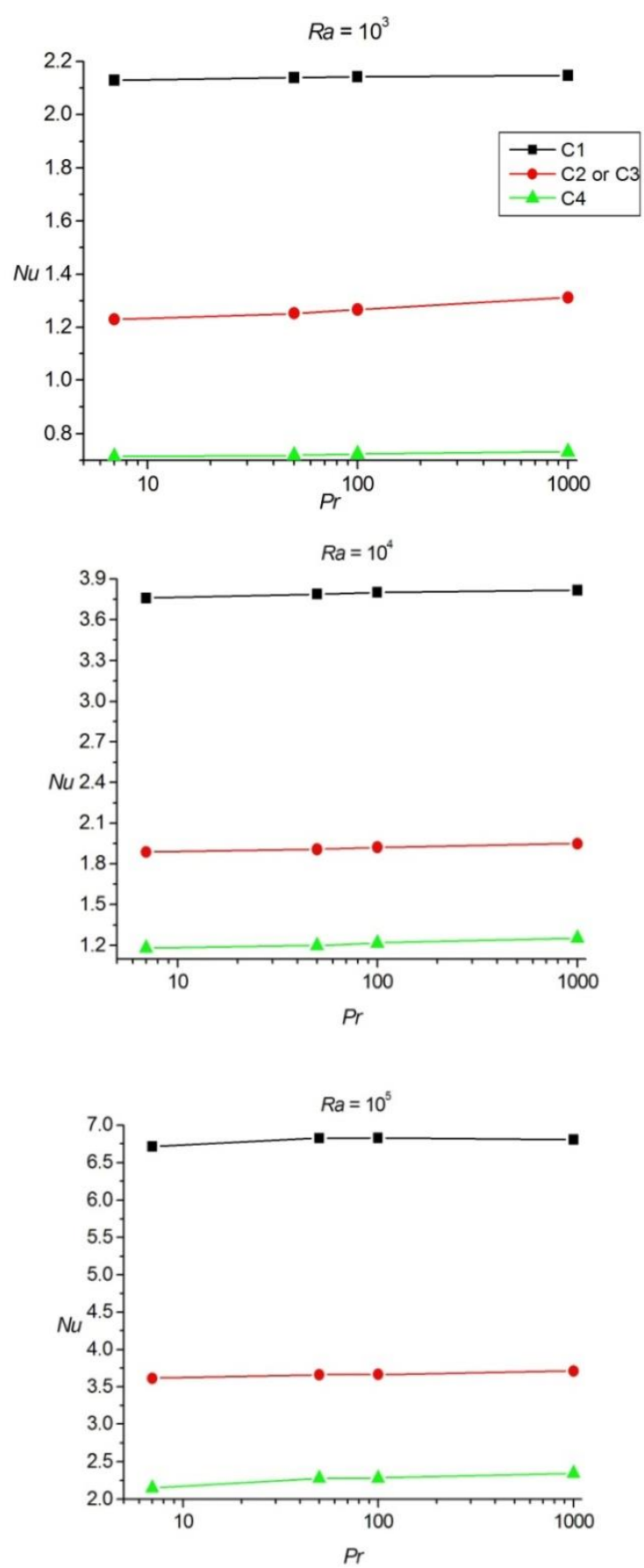

Fig. 7. Average Nusselt number versus Prandtl number for different cylinders and Rayleigh numbers

\section{REFERENCES}

1. Abu-Nada E., Masoud Z., Hijazi A.(2008), Natural convection heat transfer enhancement in horizontal concentric annuli using nanofluids, International Communications in Heat and Mass Transfer, $35,657-665$.

2. Aly A.M.(2017), Natural convection over circular cylinders in a porous enclosure filled with a nanofluid under thermo-diffusion effects, Journal of the Taiwan Institute of Chemical Engineers, 70, 88-103.

3. Arbaban M., Salimpour M.R.(2014), Enhancement of laminar natural convective heat transfer in concentric annuli with radial fins using nanofluids, Heat Mass Transfer, 47, 5181-5188.
4. Char M., Lee G.C.(1989), Maximum density effects on natural convection of micropolar fluids between horizontal eccentric cylinders, Jnt. J. Engng Sci., 36, 157-169.

5. Eid E.I. (2011) Experimental study of free convection in an elliptical annular enclosure in blunt and slender orientations, Heat Mass Transfer, 47, 81-91.

6. El-maghlany W., Abo-elazm, M., Shahata A., Eldrainy Y.(2016), Mixed convection in an eccentric annulus filled by copper nanofluide, Thermal Science, 20, 1597-1608.

7. HaM.Y., Kim J.G. (2004), Numerical simulation of natural convection in annuli with internal fins, KSME International Journal, $18,718-730$.

8. Hadidi H., Manshadi M.K.D., Kamali R.(2020), Natural convection of power-law fluids inside an internally finned horizontal annulus. Iranian Journal of Science and Technology, Transactions of Mechanical Engineering, 44, 415-425.

9. Ho C.J., Lin Y. H., Chen T.C. (1989), A numerical study of natural convection in concentric and eccentric horizontal cylindrical annuli with mixed boundary conditions. International Journal of Heat and Fluid Flow, 10, 40-47.

10. Hussein A.K.(2013), Computational analysis of natural convection in a parallelogrammic cavity with a hot concentric circular cylinder moving at different vertical locations, International Communications in Heat and Mass Transfer, 46, 126-133.

11. Iqbala Z., Syed K. S., Ishaq M.(2017), Optimum configurations of annulus with triangular fins for laminar convection, Thermal Science, 21, 161-173.

12. Kozlov N. (2018), Steady flow in an annulus with a varying number of deflectors at rotational vibration, Fluid Dynamic Research, 50, 051402.

13. Kuehn T.H., Goldstein R.J.(1976), An experimental and theoretical study of natural convection in the annulus between horizontal concentric cylinders, Journal of Fluid Mechanics, 74, 695-719.

14. Laidoudi H. (2020), Buoynacy-driven flow in annular space from two circular cylinders in tandem arrangement, Mutallurgical and Materials Engineering, 26, 87-102.

15. Laidoudi H., Helmaoui M., Bouzit M., Ghenaim A. (2020) Natural convection of Newtonian fluids between two concentric cylinders of a special cross-sectional form, Thermal Science, 00, 00-00. 10.2298/TSCI200201190L.

16. Masoumi H., Aghighi M.S., Ammar A.(2019), Laminar natural convection of yield stress fluids in annular spaces between concentric cylinders, International Journal of Heat and Mass Transfer, 138, 1188-1198.

17. Matin M.H., Khan W.A.(2013), Laminar natural convection of nonNewtonian power-law fluids between concentric circular cylinders, International Communications in Heat and Mass Transfer, 43, 112121.

18. Nada S.A., Said M.A.(2019), Effects of fins geometries, arrangements, dimensions and numbers on natural convection heat transfer characteristics in finned-horizontal annulus, International Journal of Thermal Sciences, 137, 121-137.

19. Nasiri D., Dehghan A. A., Hadian M. R.(2017), Conjugate natural convection between horizontal eccentric Cylinders, Heat Mass Transfer, 53, 799-811.

20. Pandey S., Park Y. G., Ha M. Y.(2019), An exhaustive review of studies on natural convection in enclosures with and without internal bodies of various shapes, International Journal of Heat and Mass Transfer, 138, 762-795.

21. Sheikhzadeh G. A., Arbaban M., Mehrabian M. A. (2013), Laminar natural convection of $\mathrm{Cu}$-water nanofluid in concentric annuli with radial fins attached to the inner cylinder, Heat Mass Transfer, 49, 391-403

22. Zhang H.L., Tao W.Q., Wu Q.J.(1992), Numerical Simulation of natural convection in circular enclosures with inner polygonal cylinders, with confirmation by experimental results. J. of Thermal Science, 1, 249-258. 